\title{
Entre hygiène, thérapeutique et pédagogie du corps (France, Allemagne, Angleterre): histoires européennes d'une spécialisation?
}

Grégory Quin

La croyance dans les vertus hygiéniques et/ou thérapeutiques des exercices du corps n'est pas neuve. ${ }^{1} \mathrm{Si}$ l'on retrouve déjà des traces de recommandations en termes de mouvement dans l'Antiquité chez Hippocrate ou Galien, l'émergence d'une véritable gymnastique médicale et d'un engagement plus soutenu de médecins autour de l'élaboration d'une éducation physique date du courant du XVIII ${ }^{\mathrm{e}}$ siècle, tant en Angleterre, qu'en Prusse ou en France. Le processus se poursuit au XIX ${ }^{\mathrm{e}}$ siècle sous l'impulsion de la modernisation de la médecine occidentale et s'accélère au tournant du $\mathrm{XX}^{\mathrm{e}}$ siècle, ${ }^{2}$ avant d'exploser avec les Trente Glorieuses et l'avènement d'une civilisation du loisir et de la compétition. Les débats actuels sur l'obésité infantile, sur la mobilité des personnes âgées ou sur la nécessité de se maintenir «en forme» par l'exercice physique, témoignent encore des liens très forts qui peuvent exister entre les recommandations en termes de pratiques d'exercice corporel (fussent-ils recommandés par des «auxiliaires»: kinésithérapeutes, physiothérapeutes, enseignants, éducateurs, etc.) et les savoirs de la médecine moderne (et donc des médecins qui en sont les dépositaires).

L'objet du présent essai est de mettre en regard quelques travaux issus des historiographies allemandes, anglaises et françaises pour essayer de saisir d'une part les éventuelles spécificités nationales en termes de production historique sur l'engagement des médecins autour de l'éducation physique, et d'autre part d'essayer d'esquisser les contours d'une histoire comparée

1 Je remercie Monique Schneider pour ses relectures successives et ses conseils avisés.

2 Weber 1971; Cronin 2007.

Grégory Quin, Institut des Sciences du Sport, Faculté des sciences sociales et politiques de l'Université de Lausanne et Institut Universitaire d'Histoire de la Médecine et de la Santé Publique, Lausanne (gregory.quin@unil.ch). 
notamment autour du processus de spécialisation, et de l'influence de travaux antérieurs sur l'analyse d'historiens autour de l'élaboration d'une éducation physique plus rationnelle.

Notre essai se fonde principalement sur six travaux, dont notamment trois thèses de doctorat soutenues récemment: L'investissement médical en éducation physique par Taïeb El Boujjoufi (2005); Über die Anfänge der Schwedischen Heilgymnastik in Deutschland par Julia Helene Von Schöler (2005) et Panser les deux mondes par Baptiste Viaud (2009). A ces travaux, nous ajoutons encore trois ouvrages récents: Wasser, Fasten, Luft und Licht. Die Geschichte der Naturheilkunde in Deutschland par Uwe Heyll (Campus 2006); La naissance de la kinésithérapie par Jacques Monet (Glyphe 2009) et A History of British Sports Medicine par Vanessa Heggie (Manchester University Press 2011).

Premier constat, né de recherches préalables à la rédaction de cet essai, les historiographies française, allemande et anglaise ne se ressemblent que très peu tant quantitativement que qualitativement; et elles ne peuvent, dans une certaine mesure, qu'être appareillées par deux. Si les travaux français sur les relations entre médecine et activités physiques sont les plus nombreux, ils s'associent aux travaux allemands dans la prise en compte - même relative des dynamiques de constitutions d'espaces spécifiques des pratiques d'exercice corporel (depuis le début du XIX ${ }^{\mathrm{e}}$ siècle). Les travaux français s'associent en revanche aux travaux anglais sur la prise en considération des processus de la spécialisation de la médecine moderne au $\mathrm{XX}^{\mathrm{e}}$ siècle, et surtout après la Deuxième Guerre mondiale.

\section{Entre médecine et pédagogie du corps: spécificités nationales des recherches et des processus}

Soulignés par de nombreux historiens, les voisinages et relations entre médecine et pédagogie - au cours des trois derniers siècles - ont été très différemment étudiés dans les différents espaces nationaux occidentaux (et tout particulièrement ici la France, l'Angleterre, l'Allemagne). En France, plusieurs doctorats ont porté ces dix dernières années sur les conditions d'engagement des médecins dans l'éducation physique, la gymnastique et les sports et sur les discours produits. ${ }^{3}$ Comme le note El-Boujjoufi dans sa conclusion, ces travaux soulignent que «l'investissement médical en éducation physique résulte d'arrangements sociaux à la faveur desquels il s'est

3 Monet 2003; El Boujjoufi 2005; Bohuon 2008; Bazoge 2008; Viaud 2009; Quin 2010. 
[progressivement] spécifié sous la forme d'une position collective». ${ }^{4}$ Souvent en forme de sociohistoire, ces travaux, tous plus ou moins influencés par le positionnement épistémologique de Jacques Defrance, ${ }^{5}$ témoignent également de la dette de l'historiographie française à des sociologues ou/et des philosophes comme Pierre Bourdieu ou avant lui Michel Foucault et Georges Canguilhem, qui ont irrigué et influencé durablement les recherches sur les liens entre médecine et éducation physique (et sports). Ces travaux dans leur ensemble donnent une vue assez large sur la sociogenèse de l'engagement médical autour de l'élaboration de l'éducation physique et sur l'invention d'une médecine des exercices corporels (médecine du [ou des] sport[s] pour le $\mathrm{XX}^{\mathrm{e}}$ siècle) au cours des trois derniers siècles, abordant les processus sous différents angles: constitution d'une position collective, poids des représentations sexuées dans les prises de position, légitimation de techniques de soins ou de professions «auxiliaires», etc.

Le processus n'est pas identique pour l'Angleterre ou l'Allemagne, où les recherches semblent être moins nombreuses et moins diversifiées sur ces sujets, notamment ces toutes dernières années. En Angleterre, deux recherches récentes ont sorti un certain nombre de processus de l'ombre, à l'initiative respectivement de Neil Carter et de Vanessa Heggie. Néanmoins, leurs analyses portent surtout sur le $\mathrm{XX}^{\mathrm{e}}$ siècle ${ }^{6}$ et les processus de la légitimation d'une médecine du sport en Angleterre. Et si selon Vanessa Heggie, au cours de la première moitié du $\mathrm{XX}^{\mathrm{e}}$ siècle, la médecine du sport anglaise souffre de ne pas avoir éclairci les frontières de son action et de son efficacité $e^{7}$ Neil Carter rappelant au passage que «l'historiographie des relations entre la médecine et les sports britanniques est maigre»,$-{ }^{8}$ elle va s'institutionnaliser plus concrètement après 1945, notamment avec la fondation de la British Association for Sport (and Exercise) Medicine (BAS(E)M) en 1952, ${ }^{9}$ puis à travers la mise en place d'un «Conseil des Sports» (Sports Council) en $1965 .{ }^{10}$

En Allemagne, l'histoire de la gymnastique médicale (Heilgymnastik) et de l'engagement des médecins autour de l'éducation physique n'a été l'objet que de travaux assez épars au cours des dernières décennies, comme le rappelle Julia von Schöler dans l'introduction de sa thèse, «le compte des

\footnotetext{
4 El Boujjoufi 2005, 218.

5 Defrance 1978. Du reste, Jacques Defrance a participé à tous les jurys de soutenance, lorsqu'il n'a pas dirigé lui-même les thèses précitées.

6 La contribution de Neil Carter dans le présent numéro thématique est tout à fait pionnière.

7 Heggie 2011, chapitres 2 et 3.

8 Carter 2009, 262.

9 Heggie 2010, 459.

10 Heggie 2010, 460-462.
} 
travaux sur l'histoire de la gymnastique médicale en Allemagne est extrêmement réduit». ${ }^{11} \mathrm{Du}$ reste, conformément à certaines caractéristiques «nationales» du développement des pratiques corporelles depuis deux siècles, c'est davantage autour des questions de l'hydrothérapie ou encore du naturisme que les travaux se sont dirigés, comme en témoigne l'ouvrage de Uwe Heyl ou les recherches de Jürgen Helfricht. ${ }^{12}$ Dans son ouvrage, Uwe Heyll rappelle ainsi qu'«à côté de l'eau et de la diète, le mouvement corporel était également intégré parmi les démarches des cures de von Schroth et Prießnitz» ${ }^{13}$ - deux fondateurs de l'hydrothérapie - dès les premières années du XIX ${ }^{e}$ siècle. L'auteur rappelle ensuite la prégnance de la naturopathie (Naturheilkunde) ou plus largement de l'application d'agents physiques (eau, air, pression, etc.) dans la médecine allemande, y compris face à un courant comme la «médecine biologique» au début du $\mathrm{XX}^{\mathrm{e}}$ siècle. ${ }^{14}$

L'ensemble de ces décalages nationaux, s'ils limitent quelque peu les possibilités de comparaison stricto sensu, n'empêchent pas d'esquisser des analyses croisées, notamment dans deux directions: la spécialisation dans le champ médical et les processus de la diffusion internationale de pratiques et de savoirs, ainsi que nous allons essayer de l'esquisser.

\section{La «médecine du sport»: spécialisations et spécialités}

Dans la foulée des travaux pionniers de George Rosen ${ }^{15}$ ou de Heinz Eulner ${ }^{16}$, et plus récents de Georg Weisz ${ }^{17}$ ou de Patrice Pinell, ${ }^{18}{ }^{1}$ 'une des questions centrales des travaux sur la gymnastique médicale, la kinésithérapie (physiothérapie) ou la médecine du sport a été de savoir si le processus de leur structuration correspondait à celui de la constitution d'une spécialité au sein d'un champ médical, soit un processus de «normalisation des catégories de santé et de maladie» ${ }^{19}$ et des traitements afférents, pour reprendre les termes de Vanessa Heggie; et par extension, les chercheurs ont voulu savoir si la dynamique globale de spécialisation des champs médicaux avait un impact sur la prise en compte du mouvement parmi les réflexions et les

11 Von Schöler 2005, 8.

12 Helfricht 2006.

13 Heyll 2006, 74.

14 Heyll 2006, chapitre 8.

15 Rosen 1944.

16 Eulner 1970.

17 Weisz 2006.

18 Pinell 2009.

19 Heggie 2010, 457. 
démarches hygiéniques et/ou thérapeutiques des médecins français, allemand ou anglais.

De fait, la spécialisation des champs médicaux est un processus qui s'amorce au tout début du XIX ${ }^{\mathrm{e}}$ siècle, de manière concomitante avec l'avènement en Europe de systèmes de pratiques d'exercice corporel. Mais ce processus n'est pas absolument synchrone pour tous les pays européens, et il s'amorce notamment à Paris avant Londres, Berlin ou Vienne en raison de la plus grande population de la ville, de son corps médical plus nombreux, du rapprochement récent qui s'y est joué entre médecine et chirurgie, ${ }^{20}$ et encore de la plus grande autonomie - notamment par rapport à un «champ du pouvoir» - dont jouissaient les médecins français dès les premières décennies du XIX ${ }^{\mathrm{e}}$ siècle. En outre, du point de vue du champ médical, et toujours selon Georg Weisz, la spécialisation ne correspond pas uniquement à une transformation des savoirs, telle que l'aurait décrite Michel Foucault dans La naissance de la clinique, elle relève également de «nouvelles conceptions de la rationalité administrative qui ont vu le jour dans l'Etat-nation du $\mathrm{XIX}^{\mathrm{e}}$ siècle.» ${ }^{21}$

En ce qui concerne, les travaux constitutifs du présent essai, deux processus doivent en fait être distingués: d'une part, la prise en compte des exercices du corps dans le cadre du processus de spécialisation de la médecine au cours du XIX ${ }^{\mathrm{e}}$ siècle, d'autre part, l'émergence d'une spécialité, la «médecine du sport», dans le champ médical au $\mathrm{XX}^{\mathrm{e}}$ siècle.

En France, la spécialisation précoce de la médecine va faire entrer plus franchement les questions des gymnastiques et de l'éducation physique dans les préoccupations des médecins - et notamment des orthopédistes dans les années 1830-1840 22 , là où en Allemagne, cela va davantage se jouer autour de l'installation de cures hydrothérapiques, dans le cadre de pédagogies corporelles influencées par une philosophie «naturelle» et par le courant gymnique suédois où le médecin joue un rôle de «serviteur de la nature».23

Pour la France, le travail de Jacques Monet est tout à fait significatif puisqu'il pose explicitement la question de la constitution - et donc de l'existence à un moment donné - d'une spécialité «kinésithérapique dans le champ médical français à la fin du XIX et au début du $\mathrm{XX}^{\mathrm{e}}$ siècle. Si son histoire est celle d'une «spécialisation impossible», elle rend bien compte des enjeux et des processus existant autour des relations entre médecine et activité phy-

20 Pinell 2009.

21 Weisz 2005, 37.

22 Monet 2003, chapitre 5.

23 Heyll 2006, 64 ss. 
sique, ainsi qu'entre médecins et professions auxiliaires (masseurs, gymnasiarques, etc.) et débouchant sur la création d'écoles de massage vers 1895 et de la Société de Kinésithérapie à Paris en 1900. Pourtant, dix ans plus tard, lors du Congrès international de physiothérapie de Paris en 1910, la kinésithérapie fait face à la montée de la physiothérapie qui cherche à regrouper kinésithérapie, hydrothérapie, thermalisme ou électrothérapie. Il existe alors une dynamique d'expansion et de diffusion de tous ces procédés, «ils ouvrent la voie à de vastes perspectives, dans lesquelles s'engouffrent des médecins praticiens qui appliquent toutes sortes d'agents physiques à toutes sortes de maladies» ${ }^{24}$ mais la kinésithérapie ne devient pas une «spécialité». Les liens entre médecine et éducation physique (et sports) vont alors se reconfigurer dans l'entre-deux-guerres, notamment en direction d'un champ éducatif. Selon El Boujjoufi, le milieu médical propose de modifier la formation des instituteurs et des enseignants d'éducation physique du secondaire, et parvient à créer des Instituts Régionaux d'Education Physique (IREP) en 1927. Il s'agit d'une formation d'une année après le baccalauréat pour enseigner l'éducation physique, et d'une «spécialisation» en hygiène de l'exercice pour les étudiants en médecine. ${ }^{25}$

Après 1945, Baptiste Viaud souligne que l'engagement important de l'Etat auprès du mouvement sportif va avoir des effets contradictoires, favorisant le contrôle en exigeant examens et certificats mais diminuant également l'influence et l'autonomie du champ médical face aux sportifs. ${ }^{26} \mathrm{Si}$ les années 1970 et 1980 seront assez propices à la médecine du sport - dans le cadre d'un accompagnement du sport de haut niveau -, le processus de «spécialisation» n'aboutit pas. Au sortir de son travail, Viaud parle même de «débâcle générale ${ }^{27}$ à propos de l'état de la médecine du sport en France depuis une vingtaine d'années.

De fait, la forte centralisation française est sans doute un élément distinctif pour comprendre l'engagement des médecins autour de l'éducation physique et des sports. En effet, en Angleterre ou en Allemagne, les processus sont différents. En Angleterre à la différence de la France, la fin de la Deuxième Guerre mondiale va favoriser l'émergence d'une médecine du sport plus autonomisée. Vanessa Heggie met notamment en avant la constitution de la $\mathrm{BAS}(\mathrm{E}) \mathrm{M}$ en 1952, à un moment où «la médecine du sport considérée comme une pratique holistique, recouvrant un espace qui va des blessures musculaires et squelettiques, aux conseils diététiques et jusqu'aux tests

24 Monet 2009, 346.

25 El Boujjoufi 2005, 110.

26 Viaud 2009, 309 et suivantes.

27 Viaud 2009, 353. 
génétiques (...) [qui plus est] sans maladies ou blessures spécifiques» ${ }^{28}$ ne représente pas l'espace de spécialisation le plus prometteur. Si, en Angleterre, la spécialisation de la médecine est profondément intriquée à la fondation d'hôpitaux et de services spécialisés ${ }^{29}$ dans le cas de la médecine du sport, Vanessa Heggie - qui parle d'une «spécialisation sans hôpitaux» à son sujet - souligne que c'est surtout à partir des années 1970, donc a posteriori, que les choses vont évoluer plus rapidement. En effet au milieu des années 1970, la BAS(E)M va promouvoir un nouveau modèle de cliniques de médecine du sport distinctes d'anciens services de rééducation ou d'orthopédie, et où de vrais «spécialistes» vont pouvoir exercer. ${ }^{30}$ L'institutionnalisation fonctionne alors ici un peu à rebours d'autres domaines de la médecine. En 1984, ces cliniques sont au nombre de 28, auxquelles s'ajoutent 15 cliniques privées spécialisées en physiothérapie et dédiées aux blessures sportives (sport injuries). Aboutissement d'un processus singulier, en Angleterre, la médecine du sport est reconnue comme une «spécialité» médicale à part entière dans le champ depuis le 21 février 2005. ${ }^{31}$

Si le processus général de la spécialisation est y finalement assez semblable ${ }^{32}$, au milieu du XIX ${ }^{\mathrm{e}}$ siècle, c'est en Allemagne - plus qu'en France ou en Angleterre - que les recherches en laboratoire vont émerger plus fortement. Loin du modèle des «écoles pratiques» ayant cours en France et en Angleterre - dont une conséquence est la constitution de formations très professionnalisantes -, en Allemagne, recherche et enseignement vont demeurer plus intriqués ${ }^{33}$, et cette particularité va marquer durablement la recherche biomédicale allemande. Au-delà de ces éléments structurels, la naturopathie influe très durablement sur l'engagement des médecins vers les usages curatifs et/ou hygiéniques du mouvement, au point qu'aujourd'hui environ $10 \%$ des médecins allemands possèdent une qualification spécifique dans ce domaine. ${ }^{34}$ Toutefois jusqu'à ce jour, la «médecine du sport» n'est pas reconnue comme une spécialité (Facharzt) en Allemagne; tout au plus y est-elle parfois évoquée comme une «petite spécialité», une qualification supplémentaire, comme dans le cas des formations contemporaines françaises.

28 Heggie 2010, 458.

29 Granshaw 1989.

30 Heggie 2010, 468; Heggie 2011, chapitre 5.

31 Heggie 2010, 459.

32 Weisz 2005.

33 Neville Bonner 1995, 232-239.

34 Heyll 2006, 273. 


\section{Les influences internationales}

Depuis la seconde moitié du XVIII ${ }^{\mathrm{e}}$ siècle, les pratiques d'exercice corporel occidentales se sont constituées dans le cadre d'une dynamique continentale. Entre 1775 et 1825: en Prusse avec Guts Muths (1793) et Jahn (1811), en Suède avec Ling (1813), en Suisse avec Clias (1816) ou encore en France avec le Colonel Amoros (1819) et en Angleterre avec les «sports modernes», 35 des «traditions» de pratiques d'exercice corporel voient le jour et vont s'influencer réciproquement par-delà les frontières nationales.

De tous ces courants, c'est la gymnastique suédoise qui nous intéresse au premier plan, car même si elle n'a pas été fondée par un médecin, ses principes sont directement influencés par l'anatomie et ses développements du début de XIX ${ }^{\mathrm{e}}$ siècle. Inaugurée par Per Henrik Ling au tout début du XIX ${ }^{\mathrm{e}}$ siècle, la gymnastique suédoise se diffuse ensuite à travers tous les continents. Rapidement diffusée en Allemagne (en Prusse) dès les années $1840^{36}$ - alors que le mouvement «national» - le Turnen de Jahn - connaît une disqualification temporaire après $1848^{37}$-, elle apparaît en France en $1847^{38}$ et en Angleterre en $1850^{39}$ sous la plume de Carl Auguste Georgii, lui-même ambassadeur de Lars Branting, le successeur de Ling à la tête de l'institution suédoise centrale de gymnastique.

Mais les influences se croisent, y compris entre les pays recevant la gymnastique de Ling, Julia Von Schöler mentionne ainsi l'existence d'un gymnase médical dès 1838 à Londres qui aurait été visité par un médecin allemand Hermann Eberhard Richter (1808-1876) de Dresde. Ce dernier, convaincu par sa visite de la nécessité de promouvoir une thérapeutique par le mouvement, participe alors à l'implantation de la gymnastique suédoise en Prusse, dont il vante les «fondements» anatomo-physiologiques et le caractère méthodique dans le développement des facultés corporelles. ${ }^{40}$ Les choses se mettent lentement en place, notamment en France et en Angleterre où la prégnance d'un modèle militaire et l'émergence des sports modernes, ${ }^{41}$ respectivement, ralentissent l'implantation de la gymnastique suédoise; néanmoins en Allemagne, ce courant de gymnastique génère plus précocement un certain nombre de travaux sur la gymnastique médicale dès les années 1840, et notamment ceux d'un certain Hugo Rothstein, dans lesquels le terme

35 Pfister 2003.

36 Von Schöler 2005, 49-53.

37 Pfister 2003.

38 Quin 2010, 642-644.

39 Georgii 1850.

40 Von Schöler 2005, 50.

41 Elias 1986. 
de «gymnastique médicale» apparaît pour la première fois dans la langue allemande..$^{42}$ Mais ce sont surtout deux médecins qui comptent parmi les principaux promoteurs de la gymnastique suédoise en Allemagne: Albert Constantin Neumann et Moritz Michael Eulenburg. Ces deux médecins sont particulièrement actifs après 1850 , période durant laquelle, ils vont s'attacher à modifier quelque peu les fondements de la gymnastique suédoise pour en parfaire l'efficacité proprement médicale, en y insérant notamment toutes les découvertes sur la «circulation et la résorption capillaire» des plus grands noms de la physiologie allemande du milieu du XIX ${ }^{\mathrm{e}}$ siècle. ${ }^{43}$

En France dans la seconde moitié du XIX ${ }^{\mathrm{e}}$ siècle, les exercices de gymnastique suédoise sont désignés comme une «kinésithérapie» ${ }^{44}$, littéralement une «thérapeutique par le mouvement», à la suite de la parution de l'ouvrage éponyme de Georgii. Ce dernier initie la pratique de la gymnastique suédoise à Paris, dont le premier gymnase est dirigé par Henri Meding, un médecin allemand installé à Paris et commentateur de la vie médicale de la capitale. ${ }^{45}$ Dans ces années 1850, la gymnastique suédoise s'insère dans les discours gymniques médicaux français de manière quelque peu paradoxale, dans la mesure où cette diffusion s'opère durant une période où l'efficacité de la gymnastique médicale est discutée, notamment dans le traitement des affections orthopédiques. ${ }^{46} \mathrm{Si}$ la gymnastique suédoise n'échappe pas aux discours méfiants vis-à-vis d'une croyance trop aveugle envers les pouvoirs curatifs de la gymnastique, ses fondements anatomiques et l'extrême localisation des mouvements qu'elle prône participent à la requalification de la gymnastique médicale après $1870 .{ }^{47}$ Et à la fin des années 1880 et au début des années 1890, la gymnastique suédoise «devient d'application générale», même si la tutelle médicale demeure prégnante à cause de la nécessité d'un encadrement qualifié.

Les débats changent de nature avec le $\mathrm{XX}^{\mathrm{e}}$ siècle, lorsque la question de l'accompagnement de la performance devient un objectif dicible parmi les médecins. Nous avons vu qu'en France, cela génère un intérêt certain dans l'après-guerre, mais non durable au-delà des années 1980, alors qu'en Angleterre et en Allemagne la dynamique est d'une certaine manière plus constante. ${ }^{48}$ Dans le cadre du développement de la médecine du sport en Angleterre, on peut constater également que l'«international» - entendez le

42 Von Schöler 2005, 11.

43 Von Schöler 2005, 77-81.

44 Georgii 1847.

45 Meding 1853.

46 Quin 2010, chapitre 9.

47 El Boujjoufi 2005, 42-99.

48 Bäumler/Court/Hollmann 2002; Heggie 2011. 
développement des compétitions sportives internationales - joue un rôle important. Ainsi, le Conseil des sports, fondé en 1965, va d'emblée soutenir un projet de recherche sur «les effets de l'altitude pour les athlètes préparant les Jeux Olympiques de Mexico de 1968».49

Dans le cas de l'Allemagne après la Seconde Guerre Mondiale, il faudrait établir une distinction entre les institutions dont se dote l'Allemagne de l'Ouest - qui ressemblent beaucoup aux institutions anglaises - et les institutions de l'Allemagne de l'Est ${ }^{50}$ dont l'engagement dans la politique étatique de dopage fut total. En effet, dès «la fondation du service médical sportif (...) en 1964 (..), les médecins du sport étaient intégrés dans la hiérarchie du système sportif (et non dans celle du ministère de la santé)». ${ }^{51}$ De fait, si l'on regarde plus en détails les dynamiques de constitution d'une médecine du sport en Allemagne de l'Ouest, force est de constater que les processus sont assez semblables à ceux de l'Angleterre. Dès 1950, une fédération allemande des médecins du sport (Deutscher Sportärztebund) est (re-)fondée. Dans la foulée, plusieurs congrès et journées d'études sont organisés à Cologne puis à Berlin. ${ }^{52}$ Et en 1953, l'Allemagne prend l'initiative d'organiser un congrès international à Francfort, rassemblant des spécialistes italiens, suisses, autrichiens, etc., dont les travaux sont en partie dirigés vers les questions de performance et d'entraînement des sportifs de haut niveau. En outre, dans les années 1950 et 1960, un conseil de la recherche en médecine du sport (Kuratorium für die sportmedizinische Forschung in der Bundesrepublik Deutschland, 1955) et un institut fédéral pour les sciences du sport (Bundesinstitut für Sportwissenschaft, 1969) vont structurer les recherches à la fois vers le sport performance et vers le sport de masse (et la santé publique). ${ }^{53}$ Les derniers travaux historiques, menés notamment par Michael Krueger, ${ }^{54}$ invitent cependant à la prudence, tant l'Allemagne de l'Ouest semble aussi s'être engagée - pour des raisons évidentes de géopolitique sportive - dans la diffusion et parfois dans l'organisation d'activités de dopage auprès de ses athlètes.

49 Heggie 2010, 460.

50 Spitzer 2004.

51 Spitzer 2005, 50.

52 Bäumler/Court/Hollmann 2002, 69-70.

53 Bäumler/Court/Hollmann 2002, 75-79.

54 Krüger 2009. 


\section{Conclusion}

Ce bref bilan historiographique, très partiel, souligne que de nombreux travaux sont encore à mener. De fait, le champ de recherches situé à l'interface entre un champ médical et un champ des pratiques d'exercice corporel, parfois pensé comme un champ éducatif dans son ensemble, n'a pas fini de livrer ses anecdotes et ses dynamiques. Si les rapports sont éphémères dans leurs structures, ils sont aussi extrêmement durables dans différents espaces nationaux depuis le milieu du XVIII ${ }^{\mathrm{e}}$ siècle. Dans ce cadre, l'autonomisation d'un champ sportif, la multiplication des compétitions sportives internationales et l'exacerbation d'un besoin de santé corporelle depuis plus de 50 ans ont largement concouru à amener les médecins au plus près des sportifs $(\mathrm{du}$ dimanche ou de haut niveau).

Au-delà d'éléments d'une sociohistoire (pensée autour du processus de spécialisation) ou d'une histoire internationale où des processus sont comparés entre différentes nations, et à l'instar des travaux de Natalia Bazoge ou d'Anaïs Bohuon (pour le cas de la France à la fin du XIX $\mathrm{X}^{\mathrm{e}}$ et au début du $\mathrm{XX}^{\mathrm{e}}$ siècle), la force des discours produits doit aussi encore être davantage analysée en termes de production de représentations ayant trait aux corps et aux assignations sexuelles induites. L'usage du qualificatif «hystérique» pour décrire un comportement féminin dans une publicité d'un grand fabricant d'articles de sport - publicité parue en 2005 dans les colonnes du premier numéro de L'Equipe féminine - souligne toutes les possibilités de reconversion d'un discours sur les corps féminins et ses pathologies dans l'actualité et encourage le prolongement de semblables recherches.

\section{Bibliographie}

\section{Sources}

Georgii, Carl August, Kinésithérapie, ou traitement des maladies par le mouvement selon la méthode de Ling (Paris 1847)

Georgii, Carl August, A few words on Kinesipathy, or Swedish Medical Gymnastics; the Application of Active and Passive movements to the Cure of Diseases, according to the method of P. H. Ling (Londres 1850)

Meding, Henri, Manuel du Paris medical (Paris 1853)

\section{Références}

Bäumler, Günther/Jürgen Court/Wildor Hollmann, Sportmedizin und Sportwissenschaft. Historisch-systematische Facetten (Sankt Augustin 2002)

Bazoge, Natalia, «Pour la Patrie:par le Foyer, par l’Ecole, par la Caserne». Une histoire genrée de la Ligue Girondine d'Education Physique (1888-1935), thèse de doctorat de troisième cycle de l’Université de Lyon (Lyon 2008) 
Bohuon, Anaïs, Entre santé et pathologie: discours médical et pratique physique et sportive féminine (1880-1922), thèse de doctorat de troisième cycle (STAPS) de l'Université Paris Sud 11 (Paris 2008)

Carter, Neil, "The Rise and Fall of the Magic Sponge: Medicine and the Transformation of the Football Trainer", Social History of Medicine 23 (2009) 261-279

Cronin, Mike, "Not Taking the Medicine: Sportsmen and Doctors in Late Nineteenth-Century Britain", Journal of Sport History 34 (2007) 23-35

Defrance, Jacques, La fortification des corps, essai d'histoire sociale des pratiques d'exercice corporel, thèse de doctorat de troisième cycle de l'Ecole des Hautes Etudes en Sciences Sociales (Paris 1978)

El Boujjoufi, Taïeb, L'investissement médical en éducation physique. Etude des conditions sociales de formation d'une position collective (1880-1950), thèse de doctorat de troisième cycle (STAPS) de l'Université Paris 10 Nanterre (Paris 2005)

Elias, Norbert, Sport et civilisation (Paris 1986)

Eulner, Heinz, Die Entwicklung der medizinischen Spezialfächer an den Universitäten des deutschen Sprachgebietes (Stuttgart 1970)

Granshaw, Lindsay, "FFame and Fortune by Means of Bricks and Mortar': the Medical Profession and Specialist Hospitals in Britain, 1800-1948”, in; Lindsay Granshaw/Roy Porter (éds.), The Hospital in History (Londres 1989) 199-220

Heggie, Vanessa, “A Century of Cardiomythology: Exercise and the Heart c. 1880-1980”, Social History of Medicine 23 (2009) 280-298

Heggie, Vanessa, "Specialization without the Hospital: The Case of British Sports Medicine", Medical History 54 (2010) 457-474

Heggie, Vanessa, A History of British Sports Medicine (Manchester 2011)

Helfricht, Jürgen Vincenz Priessnitz (1799-1851) und die Rezeption seiner Hydrotherapie bis 1918: Ein Beitrag zur Geschichte der Naturheilbewegung (Husum 2006)

Heyll, Uwe, Wasser, Fasten, Luft und Licht. Die Geschichte der Naturheilkunde in Deutschland (Francfort 2006)

Krüger, Michael, «60 Jahre Sport in Deutschland. Ein Essay zur deutsch-deutschen Sportgeschichte aus Anlass des 60. Geburtstags der Bundesrepublik», Sportwissenschaft 39 (2009) 237-250

Monet, Jacques, Emergence de la kinésithérapie en France à la fin du XIX $X^{e}$ et au début du XX siècle. Une spécialité médicale impossible. Genèse, acteurs et intérêts de 1880 à 1914, thèse de doctorat en sociologie de l'Université de Paris 1 Sorbonne (Paris 2003)

Monet, Jacques, La naissance de la kinésithérapie (Paris 2009)

Neville Bonner, Thomas, Becoming a Physician. Medical Education in Britain, France, Germany, and the United States, 1750-1914 (New York 1995)

Pinell, Patrice, «La genèse du champ médical: le cas de la France (1795-1870)», Revue française de sociologie 50 (2009) 315-349

Pfister, Gertrud, "Cultural Confrontations: German Turnen, Swedish Gymnastics and English Sport - European Diversity in Physical Activities from a Historical Perspective", Culture, Sport, Society 6 (2003) 61-91

Quin, Grégory, Le mouvement peut-il guérir? Histoire de l'engagement des médecins français dans l'élaboration de l'éducation physique (1741-1888), thèse de doctorat en histoire de l'Université de Lausanne (Lausanne 2010)

Rosen, George, The Specialization of Medicine with Particular Reference to Ophtalmology (New York 1944)

Spitzer, Giselher, Doping in der DDR. Ein historischer Überblick zu einer konspirativen Praxis (Cologne 2004)

Spitzer, Giselher, «Approche historique du dopage en République démocratique allemande: description et analyse d'un système de contraintes étatiques», Staps 70 (2005) 49-58

Viaud, Baptiste, Panser les deux mondes. Médecines et sports, entre principes hippocratiques et performances, thèse de doctorat de troisième cycle de l'Université de Nantes (Nantes 2009)

Von Schöler, Julia Helene, Über die Anfänge der Schwedischen Heilgymnastik in Deutschlandein Beitrag zur Geschichte der Krankengymnastik im 19. Jahrhundert, thèse de doctorat en médecine de l’Université de Münster (Münster 2005) 
Weber, Eugen, "Gymnastics and Sports in Fin-de-Siècle France: Opium of the Classes?", The American Historical Review 76 (1971) 70-98

Weisz, Georg, «Naissance de la spécialisation médicale dans le monde germanophone», Actes de la recherche en sciences sociales 156-157 (2005) 41

Weisz, Georg, Divide and Conquer: A Comparative History of Medical Specialization (Oxford 2006) 